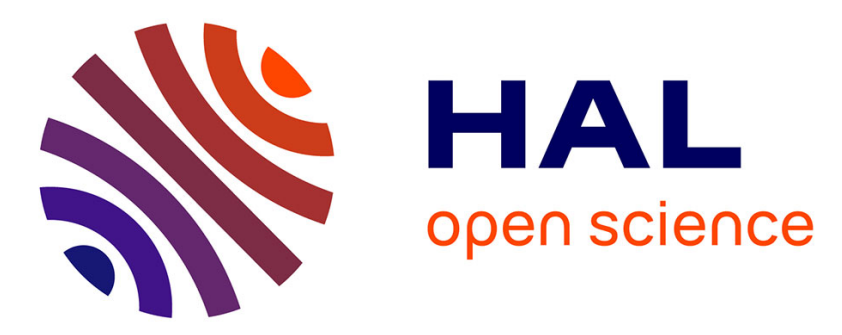

\title{
A comparison of DIC and grid measurements for processing spalling tests with the VFM and an 80-kpixel ultra-high speed camera
}

Dominique Saletti, Pascal Forquin

\section{- To cite this version:}

Dominique Saletti, Pascal Forquin. A comparison of DIC and grid measurements for processing spalling tests with the VFM and an 80-kpixel ultra-high speed camera. The European Physical Journal. Special Topics, 2016, 225 (2), pp.311-323. 10.1140/epjst/e2015-77777-x . hal-01941056

\section{HAL Id: hal-01941056 \\ https://hal.science/hal-01941056}

Submitted on 3 Dec 2018

HAL is a multi-disciplinary open access archive for the deposit and dissemination of scientific research documents, whether they are published or not. The documents may come from teaching and research institutions in France or abroad, or from public or private research centers.
L'archive ouverte pluridisciplinaire HAL, est destinée au dépôt et à la diffusion de documents scientifiques de niveau recherche, publiés ou non, émanant des établissements d'enseignement et de recherche français ou étrangers, des laboratoires publics ou privés. 


\title{
A comparison of DIC and grid measurements for processing spalling tests with the VFM and an 80-kpixel ultra-high speed camera
}

\author{
D. Saletti ${ }^{1}$, P. Forquin ${ }^{1}$ \\ ${ }^{1}$ Univ. Grenoble Alpes, 3SR, F-38000 Grenoble, France
}

Shortened version of the title:

Comparison of DIC and grid method applied to VFM for spalling tests

\begin{abstract}
.
During the last decades, the spalling technique has been more and more used to characterize the tensile strength of geomaterials at high-strain-rates. In 2012, a new processing technique was proposed by Pierron and Forquin [1] to measure the stress level and apparent Young's modulus in a concrete sample by means of an ultra-high speed camera, a grid bonded onto the sample and the Virtual Fields Method. However the possible benefit to use the DIC (Digital Image Correlation) technique instead of the grid method has not been investigated. In the present work, spalling experiments were performed on two aluminum alloy samples with HPV1 (Shimadzu) ultra-high speed camera providing $1 \mathrm{Mfps}$ maximum recording frequency and about 80 kpixel spatial resolution. A grid with $1 \mathrm{~mm}$ pitch was bonded onto the first sample whereas a speckle pattern was covering the second sample for DIC measurements. Both methods were
\end{abstract}


evaluated in terms of displacement and acceleration measurements by comparing the experimental data to laser interferometer measurements. In addition, the stress and strain levels in a given cross-section were compared to the experimental data provided by a strain gage glued on each sample. The measurements allow discussing the benefit of each (grid and DIC) technique to obtain the stress-strain relationship in the case of using an 80-kpixel ultra-high speed camera.

Keywords: full-field measurements; high strain rate; high speed imaging; spalling tests; grid method; DIC; virtual fields method.

\section{Introduction}

The spalling technique is commonly used to investigate the dynamic tensile strength of brittle geomaterials (concrete, high strength concrete, rock) at strain rates ranging from a few tens of /s to about 200 /s. The experimental set-up consists in launching a short cylindrical projectile with a gas gun facility against a Hopkinson Pressure Bar, put in contact with the specimen. A dynamic tensile loading is created into the specimen by the reflection of a short compressive pulse at the free end of the specimen. Then, the tensile failure of the tested material can be analysed [2, 3, 4]. In the literature, spalling tests have been used to study the Dynamic Increase Factors (DIFs: ratio of dynamic strength to quasi-static strength) $[4,5,6,7]$ of concrete in tension, revealing its sensitivity to the applied strain rate.

This set-up was first used in its present form a century ago [8]. The post-processing of the test realised in 1923 was related to the velocity of ejection of the fragments linked to the distance they crossed, upon basic kinematic hypothesis. The technological improvement of measurement techniques during the last 30 years has increased the number of studies using this test method to analyse the mechanical properties of 
concrete-like materials. Among the techniques used to identify the maximal tensile strength of the specimen during a spalling test, the Novikov's method prevails $[9,2,4]$ : it is based on the measurement of the velocity profile on the rear face of the sample. However, it relies on the assumption of a linear-elastic behaviour of the tested material until the stress-peak and provides only its spall strength. No information on the softening behaviour that follows is available. Recently, Pierron and Forquin [1] introduced the use of a full-field measurement technique, the grid method, in the spalling tests of concrete. A Virtual Field Method (VFM) was associated to these measurements in order to get the stress field in the region of interest captured by an Ultra-High-Speed (UHS) camera. This was the first use of the full-field measurement in a spalling test. Digital Image Correlation (DIC) is an alternative technique to grid method to measure displacement fields at the surface of the specimen. This technique is widely used nowadays and is quite easy to be set by the application of a painted speckle. However, it can be really challenging to use DIC in the spalling test conditions. The UHS cameras currently available at recording frequency of one million frames per second (Mfps) present low resolutions (around a hundred of kpixels) and DIC measurement is strongly dependent of this parameter. Nevertheless, as the improvement of UHS cameras is expected, the use of DIC in spalling tests has to be investigated as from now. The aim of this study is to present a comparison between the performance of the grid method and the DIC technique in the evaluation of the stress, strain, acceleration and displacement in the specimen during a spalling test, after combining each full-field measurement to the Virtual Field Method. For this purpose, spalling tests were performed on an aluminium alloy. Two specimens were used: one with a grid glued on its surface and another with a painted speckle on its surface. The UHS camera Shimadzu HPV-1 (312 x 260 pixels $\left.^{2}\right)$ was used at a recording frequency of 500'000 and 1'000'000 frames per second. Performances and limitations of this camera have been first evaluated in previous works $[10,11]$ by using the grid method. The HPV-1 camera being 
the first in-situ storage image sensor UHS camera (ISIS architecture: single CCD sensor with embedded memory storage) providing a resolution of about 80 kpixels at framerate up to $1 \mathrm{Mfps}$, the present study aims to constitute a benchmark regarding the new cameras coming onto the market at this moment.

\section{Experimental technique}

\section{Samples and spalling test technique}

The material tested in this study is a high-strength aluminum, also used for the projectile and the Hopkinson bar of the spalling testing set-up and is characterized by a 1D wave speed C of $5090 \mathrm{~m} / \mathrm{s}$, a density of $2810 \mathrm{~kg} / \mathrm{m}^{3}$ and a Young's modulus equal to $72.8 \mathrm{GPa}$ [4]. The samples are cylindrical, $45 \mathrm{~mm}$ in diameter and $140 \mathrm{~mm}$ in length for the one with a grid and $100 \mathrm{~mm}$ for the one with a painted speckle. These dimensions are summarized in the Figure 1. This difference in length has no influence on the results of the study: there is no influence on the level of uniaxial stress in the specimen as the sample is expected to have an elastic behavior and the observation window used with the UHS camera, starting from the free end of the specimen, has the same size for both techniques. Both samples are instrumented with a $20 \mathrm{~mm}$-long strain gauge placed at 50 $\mathrm{mm}$ from the rear face (the free end).

The spalling test set-up used in this study is composed of a $50 \mathrm{~mm}$-long spherical-capended projectile and a Hopkinson bar both with a diameter of $45 \mathrm{~mm}$. An illustration of the experimental set-up is given on Figure 2. A short incident compressive pulse is created and propagates through the Hopkinson bar. Then, the pulse is transmitted to the sample and reflects at the free-end of the specimen as a tensile pulse leading to a dynamic tensile loading within the sample. The particle velocity at the rear-face is 
recorded with a laser interferometer from Polytec, enabling velocity measurements up to $20 \mathrm{~m} / \mathrm{s}$ with a $1.5 \mathrm{MHz}$ bandwidth [4].

\section{Optical measurement}

A Shimadzu HPV-1 UHS camera was used in this study and described by Pierron and Forquin [1]. This device allows recording a sequence of 102 images up to one million frames per second (fps) with a constant resolution of $312 \times 260$ pixel $^{2}$. Two frame rates were employed: $1 \mathrm{Mfps}$ and $500 \mathrm{kfps}$. For all the tests, the exposure time was set to $0.5 \mu \mathrm{s}$. The horizontal axis of the image corresponds to the longitudinal axis of the specimen and the left-hand side of the image corresponds to the free-end of the specimen (Figure 1). For the sake of comparison, the same image scale (about $0.2 \mathrm{~mm}$ per pixel) was set for both full-field measurement techniques. The length of the specimen capture by the camera is around $60 \mathrm{~mm}$. Table 1 summarizes the different settings used in this paper.

\section{Grid Method}

For the grid method, the magnification was adjusted to get a 5-pixels grid spacing. The pitch of the grid being $1 \mathrm{~mm}$, the image scale is $0.2 \mathrm{~mm}$ per pixel so the length of the specimen captured by the optical device is around $60 \mathrm{~mm}$. This observation window with the grid can be seen on Figure 1(b), for a reference image. The displacement fields are computed in the same way as in Pierron and Forquin [1], thanks to a characterisation by phase modulation, considering the local intensity and the contrast produced by the grid on the image. As it was found in this study, a significant low-pass filtering should be used to extract the strain values. This is due to the fact that the amplitude of displacement is small (less than half a millimetre) leading to displacement maps with high noise content. Hence, a diffuse approximation is processed. This filtering procedure is a local fit of a second-degree polynomial using specific weighing function 
(more details can be found in [12]). As a full description of the grid method would overload the content of this paper, the authors also invite the reader, to complete the description, to refer to $[13,14]$. More information on the metrological performance of the HPV1 ultra-high speed camera for full-field deformation measurements with the grid method are also given in [11].

\section{Digital Image Correlation}

The DIC (Digital Image Correlation) method has been widely used until now to investigate the mechanical response and damage behaviour of materials. In this study, the Correli ${ }^{4}$ code was used to measure the displacement and strain fields. The principle of this software is given by Besnard et al [15]. A Region of Interest (ROI) is defined on a reference image and a mesh grid is created with a user-defined element size. The elements of this mesh (with Q4-shape functions) are also called Zone of Interest (ZOI). Based on a global approach, each element is dependent on the surrounding ones. The correlation computation consists of finding a displacement field $u$ to link an image of the test sequence, the deformed image, $g$, to the reference image, $f$. The strain field is then computed on the surface of the specimen using the gradient of the displacement field. Hence, black and white paints were applied at the surface of the specimen, creating a heterogeneous speckle, as it can be seen on Figure 1(a). The quality of the speckle and the settings of the camera were validated by an image analysis taking into account the histogram of the image. For this study, the image scale was set to $0.196 \mathrm{~mm} / \mathrm{px}$, close to the magnification set with the grid method. All the DIC computation results presented in this paper were obtained with a ZOI size of $8 \mathrm{px}$ (around 1.5 times the pitch of the grid).

\section{The Virtual Field Method (VFM)}

The Virtual Field Method is based on the principle of the virtual work. It was successfully used for processing the experimental data of a spalling test in association 
with the grid method [1]. One advantage of this technique is that it can be used with any full-field measurement technique that measures kinematic fields.

Before using this method, the first step lies on the double differentiation of the displacement fields. A temporal fitting is applied on this result with a second-order polynomial function over a sliding of five images for measurements obtained with DIC and seven images for measurements obtained with the grid method. This results in an acceleration map required for the computation of the stress along the specimen with the Virtual Field Method.

As described in Pierron and Forquin [1], by defining in this case a rigid body-like virtual field, the mean stress in any cross-section $S_{x}$ of coordinate $x$ along the length of the sample filmed by the camera can be calculated according to Eq. 1:

$$
\overline{\sigma_{x x}(x, t)}=-\rho b(x) \overline{a_{x}(x, t)}
$$

Where $t$ corresponds to the current time, $\overline{\sigma_{x x}}$ denotes the mean axial stress in the cross section $S_{x}$ of coordinate $x, \rho$ is the mass per unit volume of the sample, $b(x)$ corresponds to the length between the cross-section $S_{x}$ and the free-end, and $\overline{a_{x}(x, t)}$ corresponds to the mean acceleration between the cross-section $S_{x}$ and the free-end.

Finally, the stress-strain curves in cross section $S_{x}$ can be obtained by combining the axial stress measurements with the axial strain $\varepsilon_{x x}(x, t)$, measured with DIC or grid method.

\section{Measurement results and discussion}

In order to have a comparison as relevant as possible, the authors tried to perform all the spalling tests with the same kinetic input energy. The amplitude of input loading can be evaluated by comparing the velocities measured with the laser interferometer at the 
free-end of the specimen and reported on Figure 3. The maximum rear face velocity varies between $9.7 \mathrm{~m} / \mathrm{s}$ and $12.5 \mathrm{~m} / \mathrm{s}$. The black lines refer to the experiments performed with the grid method and the grey lines correspond to the tests conducted with the DIC method. The two different time periods of the curves are related to the different lengths of specimen.

In the next sections, the results provided by both full-field measurement techniques (grid and DIC) are compared to the results obtained from independent measurement systems. In a first part, the kinematic fields are compared to the laser interferometer measurements. In a second part, the strain field measurements and the stress values along the specimen obtained with the VFM are compared to measurements from the strain gauges glued on the samples.

\section{Comparison with the laser interferometer measurement}

\section{Displacement}

The displacement on the free-end is obtained from the laser measurement by single time-integration of the particle velocity. On Figure 4, a comparison is made with the displacement given by the full-field measurements.

In the case of the grid method, the displacement of the free end of the specimen corresponds to the mean axial displacement along the first transversal line of the grid. In the case of DIC computations, the displacement is not directly measured at the free end of the specimen. This is due to the fact that, once the ROI is selected for the computation, the software creates a mesh inside this ROI with margins. So, the displacement is evaluated at $2.5 \mathrm{~mm}$ from the free-end (ZOI size of $8 \mathrm{px}$ ). Nevertheless, this distance to the free-end is supposed to be small enough to have no influence on the evaluation of the displacement at the free end. As for the grid method, the displacement is averaged along the first transversal line of the mesh. 
Figure 4 presents the displacement as function of time measured in each test. Despite the fact that the displacement measurement is not evaluated exactly at the free-end of the specimen, the DIC measurements appear to be very close to the interferometer ones. Both measurements performed with the grid and DIC methods seem to be in good agreement with those obtained from the laser interferometer. However, for the GRID-02 test, the measurement presents a noise perceptibly higher than in the three other tests, resulting in a displacement measurement significantly different from the standard laser one. A summary of the deviation of measurement is presented in the Table 2 . Comparisons have been done for two values of displacement: $0.1 \mathrm{~mm}$ and $0.2 \mathrm{~mm}$. This range of value is consistent with the displacement measurement usually found for brittle such as concrete in spalling test. An evaluation has also been made for interval of displacement of $0.2 \mathrm{~mm}(0.05 \mathrm{~mm}$ to $0.25 \mathrm{~mm})$, in order to analysis the variation of the difference between the laser interferometer values and the one with digital image methods. A mean value (in \%) is presented for each test and the standard deviation is also given. The DIC technique seems to give better results than the grid method regarding displacement. The deviations from the laser measurements can be partially explained by the noisy content of the image, due to the UHS camera. However, in the case of GRID-02 test, the important gap reported in Table 2 can be also related to the sensitivity of the grid method. Indeed, if 5-pixels grid spacing is not correctly set for the image, a slight difference may lead to an important deviation of the measurement. This is not the case of DIC, which explain why the difference between DIC-01 and DIC-02 are not as important as in the grid method case.

\section{Acceleration}

As the acceleration maps are used to evaluate the stress in the specimen with the virtual field method (Eq. 1), it is important to assess the quality of the acceleration measurements with both full-field measurement methods (Grid and DIC). Acceleration is 
first calculated by time-deriving the particle velocity measurement of the laser interferometer. Regarding the optical measurement, accelerations are computed as described in the previous section, by temporal fitting of a second-order polynomial function over a number of images depending on the full-field measurement technique: five images are used for the DIC and seven for the grid method. The acceleration is evaluated for each image by spatial-averaging the acceleration values on each node along the closest transversal edge to the free-end. As for displacement, accelerations with the DIC method are not really evaluated at the free-end of the specimen but at $2.5 \mathrm{~mm}$ from the free-end.

The results are presented in the Figure 5. First, the magnitude and shape of the acceleration in all tests (DIC-01, DIC-02, GRID-01 and GRID-02) are quite consistent with the interferometer measurements. The discrepancy observed for GRID-02 can be linked to the noise observed in the displacement measurement. It is observed that the results provided by the grid method seem to be noisier than the ones obtained from the DIC method. As for displacement, a comparison between the laser interferometer and the optical measurements have been done and presented in the Table 3.

Apart from the fact that the light level might have slightly changed between DIC-01 and DIC-02 tests, the frame rate used for the two tests seems to have an influence on the results. Inter-frame time between two images was set to $1 \mu$ s for DIC-01 and $2 \mu$ s for DIC-02 with the same time exposure $(0.5 \mu \mathrm{s})$. This effect can also be observed between GRID-01 and GRID-02, in a less significant way. With the DIC method the sensitivity to the variation of light at $1 \mu$ s (due to the technology of the sensor of HPV1 camera) seems to have more influence on the results. Finally, regarding the level of noise in acceleration measurement (Figure 5), one may ask whether the acceleration map and the virtual field method can be employed for measuring the stress in the sample (Eq. 1). In fact, the 
spatial-averaging of the acceleration over the length $b$ according to the Equation (1) reduces the noise observed here.

\section{Comparison with strain gauge measurements}

Strain gauges provide a measurement of the strain in a local area of the specimen. For both specimens, a strain gauge is located at $50 \mathrm{~mm}$ from the free-end and delivers a measurement of the longitudinal strain that is assumed to be homogeneous over the cross-section of the specimen. By making the assumption of an elastic behaviour of the specimen during the test, the stress at this location can also be evaluated.

In the next section, a comparison of experimental results is proposed over a period of time about $80 \mu$ s corresponding to the time interval between the beginning of the test to the beginning of the second compressive phase.

\section{Strain measurement}

As with strain gauge measurements, the strain values obtained from DIC and grid techniques were averaged along the transverse direction of the specimen and over a zone of length $22 \mathrm{~mm}$ for DIC and $20 \mathrm{~mm}$ for the grid method corresponding to the position of the gauge.

The results are presented in the Figure 6. As for acceleration, the change of strain measured by DIC and grid methods is similar to the data obtained from the strain gauges. Regarding the maximum value of strain in compression, overestimations of $34 \%$ for GRID-01 and $42 \%$ for DIC-01 are reported. In the tensile stage, which is of greater interest in the spalling tests, a difference of $17 \%$ for GRID- $01,18 \%$ for GRID-02, $14 \%$ for DIC-02 is reported compared to the strain gauges results. Considering visually the results on the whole process, the grid method may produce noisier measurement than the DIC method, especially in the case of low contrast or wrong spatial scaling adjustment. For instance in GRID-02 test, a part of the noise is supposed to comes from a weaker grey-level dynamic of images that increases the displacement deviation 
(Figure 4d). Otherwise, if GRID-02 experiment is not considered, according to the values reported in Table 4, the errors made in the measurement of strain by the two techniques are of the same magnitude.

\section{Stress measurement}

For a uniaxial stress-state in the sample and considering an elastic behaviour of the sample the stress can be evaluated in the cross-section at the strain gauge location by:

$$
\sigma_{x x}(x, t)=E \varepsilon_{x x}(x, t)
$$

Where $\sigma_{x x}$ is the uniaxial stress along the x-axis and $\varepsilon_{x x}$ the uniaxial strain along the xaxis and measured by the strain gauge.

As described in the previous section, the stress in the same cross-section can be evaluated with the full-field measurement technique (Eq. 1). By applying the Virtual Field Method to the kinematic fields, the stress can be evaluated for each cross-section of the specimen included in the observation window visualised with the camera. To make the comparison with the strain gauge measurement as relevant as possible, the stress is calculated by averaging the local stress in a zone of length of $22 \mathrm{~mm}$ for DIC and $20 \mathrm{~mm}$ for the grid method. Figure 7 reveals the same conclusion than for the strain: the shapes of the curves are consistent with the strain-gauges measurements.

Regarding the maximum stress values in the compression stage, the difference between the strain gauge and the optical measurements are: $18.4 \%$ for GRID-01, $28 \%$ for GRID$02,44 \%$ for DIC-01, $13.2 \%$ for DIC-02. For the tensile stage, the deviation between the strain gauge and optical measurements are: $25.8 \%$ for GRID-01, $30.4 \%$ for GRID-02, $5.5 \%$ for DIC-01 and $20 \%$ for DIC-02. These values are obtained by analysing the maximal values obtained in each phase (compression and tension), and constitute a first estimate of the error that could be made by evaluating the spall strength of materials 
with this experimental processing. The corresponding stress deviations (mean value and standard deviation) are reported for each phase (compression and tension) in the Table 5. As it was expected, the stress curves present less noise than in the case of the "spot measurement" of acceleration (Figure 5) as the spatial-averaging of the acceleration to evaluate the stress (Eq. 1) constitutes a filter of the data. According to the values of deviation in the tensile phase, (of greater interest for testing the tensile strength of geomaterials), one can see that the differences obtained with grid method and DIC are quite similar if GRID-02 is not considered (12.3 Mpa for GRID-01 compared to $16.8 \mathrm{MPa}$ and 13.0 MPa for DIC).

\section{Stress-strain curves}

The last point of comparison available with the measurements conducted in this study is the production of a stress-strain curve. Figure 8 presents for each test the curves obtained with the VFM applied to the kinematic measurements. The plots of the strain gauge data are straight lines, due to the linear assumption of an elastic behaviour (Eq. 2).

GRID-02 is clearly different from the expected shape. This is mostly due to the poor quality of displacement and strain measurements presented in Figure 6d and Figure 6d. For GRID-01, DIC-01 and DIC-02, the gap observed from the expected linear behaviour can be both explained by the error in the measurement of the stress and of the strain with the grid and DIC methods. This effect is emphasised for DIC-01 test. In order to appreciate theses results, Young modulus has been evaluated from these curves with ordinary least-squares (OLS) estimation and the results are presented in the Table 6. The difference with the standard Young Modulus value of $72.8 \mathrm{GPa}$ is established. If GRID-02 is not considered, an error between $10 \%$ and $15 \%$ is obtained. 


\section{Conclusion}

In this study, the authors attempt to make a comparison between the use of the grid method and the digital image correlation method in the Virtual Fields Method to postprocess a spalling test. The results are compared to reference values, which are obtained with a laser interferometer regarding displacement and acceleration, and obtained with a strain gauge glued on the sample regarding strain and stress. This approach was used to minimize the bias due the fact that it was not possible to apply the grid method and the DIC method for a same test on a same specimen. So, these techniques were assumed to be a standard for this study and quantitative comparison has been proposed. The level of quality of the full-field measurements produced in this study is partly linked to the quality of the CCD sensor of the Shimadzu-HPV1 UHS camera. Nevertheless, at the time of the study, only few cameras can reach the same level of performance and the fact that the same camera with the same settings was used for all the tests reduces a potential bias from this point of view.

By considering all the comparisons made in this study, the advantages and the drawbacks of each method can be listed. First, the grid method is a technique that is dependent from the adjustment of the spatial scaling of the image to an odd number of pixels per grid pitch. Consequently, the grid method may be sensitive to a bad adjustment of the pixel spacing of the grid. On the other hand, the results obtain with the DIC are more stable from a test to another one. Moreover the setting time is considerably decreased with the DIC method and it provides flexibility for the choice of the window size. On the other hand, solving contrast and spatial scaling problems, noise obtained with the DIC and grid methods seems to be similar as for the measurement of strain and displacement than for the local measurement of acceleration (Table 2).

Nevertheless, the DIC method is sensitive to the resolution of the image and the size of the zone of interest has a great influence on the quality of the results. In this study, a ZOI 
of 8 -by-8 pixels ${ }^{2}$ has been applied in order to be in accordance with 5-pixel grid spacing of the grid method. The results may be improved by increasing the size of the ZOI but the low definition of the image obtained by the 80 -kpixels-UHS camera limited this possibility in the present study. The grid method appears, in that case, to have a much better spatial resolution. Another point is that to perform the DIC processing, a mesh is created on this image. In CorreliQ4, the margin employed to create this mesh leads to a loss of information (as an example, the displacement at the free-end of the sample was impossible to obtain). This is not the case for the grid method for which the grid is directly printed on the surface of the specimen.

Regarding the accuracy in the measurement of the stress (Table 5), which is of a great interest for the spalling test, the performances of the DIC and the grid method are quite similar and it cannot be concluded there is an interest into choosing a technique instead of another in the case of the measurement of small strain gradients. In the future, the increase of the spatial resolution of ultra-high speed imaging at frame-rates up to few Mfps could more favour the DIC technique over the grid method.

\section{Acknowledgements}

This work has been partially supported by the LabEx Tec 21 (Investissements d'Avenir grant agreement $\left.\mathrm{n}^{\circ} \mathrm{ANR}-11-\mathrm{LABX}-0030\right)$

\section{References}

[1] F. Pierron, P. Forquin, Strain 48, 388 (2012)

[2] H. Schuler, C. Mayrhofer, K. Thoma, Int. Journal Impact Eng. 32, 1635 (2006)

[3] J. Weerheijm, J.C.A.M. van Doormaal, Int. J. Impact. Eng. 34, 609 (2007)

[4] B. Erzar, P. Forquin, 2010. Exp. Mech. 50, 941 (2010) 
[5] J.R. Klepaczko, A. Brara, Int. J. Impact Eng. 25, 387 (2001)

[6] B. Erzar, P. Forquin, Mechanics of Materials 43, 505 (2011)

[7] B. Erzar, P. Forquin, Int. J. Solids Struct. 51, 2559 (2014)

[8] J.W. Landon, H. Quinney, Proc. R. Soc. 103, 622 (1923)

[9] S.A. Novikov, I.I. Divnov, A.G. Ivanov, Fizika Metallov i Metallovedeniye 21, 608 (1966)

[10] F. Pierron, R. Cheriguene, P. Forquin, R. Moulart, M. Rossi, M. Sutton, Applied Mech. Mat. 70, 81 (2011)

[11] M. Rossi, F. Pierron, P. Forquin, Meas. Sci. Technol 25, 025401 (2014)

[12] S. Avril, P. Feissel, F. Pierron, P. Villon, Meas. Sci. Technol. 17, 857 (2008)

[13] Y. Surrel, in Interferometry '94: Photomechanics, Warsaw (Poland), 1994, edited by R.J. Pryputniewicz and J. Stupnicki (SPIE), p. 118

[14] S. Avril, E. Ferrier, P. Hamelin, Y. Surrel, A. Vautrin, Composite Part A 35, 873 (2004)

[15] G. Besnard, F. Hild, S. Roux, Exp. Mech. 46, 89 (2006) 


\section{Figure captions}

Figure 1. Description of the two specimens used for the study. (a) Reference image used for DIC computations with a painted speckle pattern. (b) Reference image used for the grid method computations. (c) Dimensions of the specimen. Dimensions are given in $\mathrm{mm}$.

Figure 2. Experimental device for spalling tests. (The ultra-high-speed camera is not represented).

Figure 3. Velocity measured at the free-end of the specimen for the four tests.

Figure 4. Displacement measurement with laser interferometer and the DIC $(a, b)$ or the grid method (c, d).

Figure 5. Acceleration measurement with laser interferometer and the DIC $(a, b)$ or the grid method (c, d).

Figure 6. Strain measurement at $50 \mathrm{~mm}$ from the free-end of the specimen. Comparison between values from strain gauges and the DIC $(a, b)$ or the grid methods $(c, d)$.

Figure 7. Stress measurement at $50 \mathrm{~mm}$ from the free-end of the specimen. Comparison between the strain gauge measurements and the one obtained with VFM associated to the DIC $(a, b)$ or the grid method $(c, d)$. 
Figure 8. Strain-stress curves at $50 \mathrm{~mm}$ from the free-end of the specimen. Comparison between strain gauge measurements and the one obtained with VFM associated to DIC (a, b) or grid method (c, d). 


\section{Table captions}

Table 1. Summary of the test campaign (DIC: Digital Image Correlation).

Table 2. Differences (\%) in the measurement of the displacement between the laser interferometer and DIC and grid techniques. (STD: standard deviation).

Table 3. Differences (\%) in the measurement of the acceleration between the laser interferometer and DIC and grid techniques. (STD: standard deviation).

Table 4. Differences (\%) in the measurement of the strain at $50 \mathrm{~mm}$ from the free-end of the specimen between the gauge and DIC and grid techniques. (STD: standard deviation).

Table 5. Difference (MPa) between the measurement of the stress at $50 \mathrm{~mm}$ from the free-end of the specimen between the gauge and DIC and grid techniques. (STD: standard deviation).

Table 6. Young modulus values identified with an ordinary least-squares evaluation. 
(a)

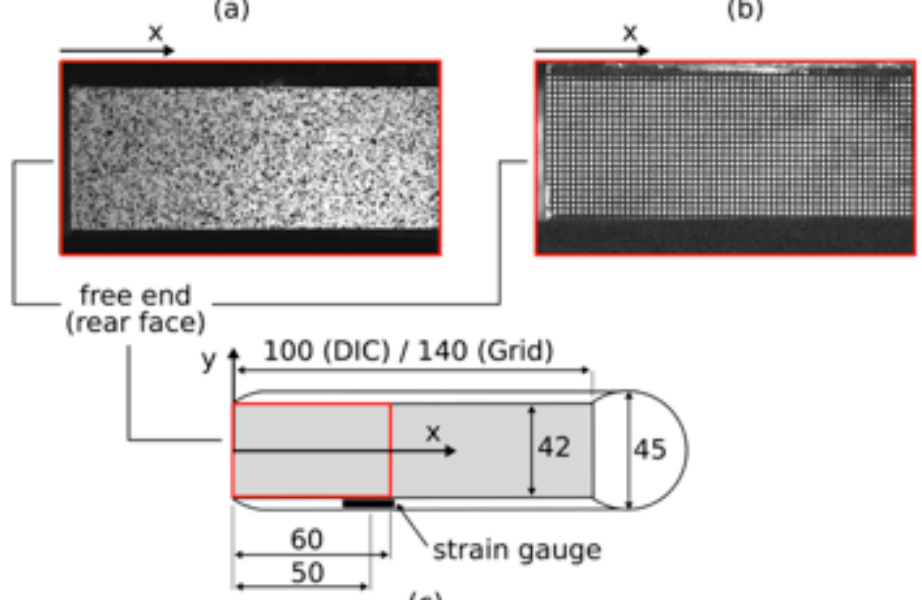

(c)

Figure 1: Description of the two specimens used for the study. (a) Reference image used for DIC computations with a painted speckle pattern. (b) Reference image used for the grid method computations. (c) Dimensions of the specimen. Dimensions are given in $\mathbf{m m}$. 


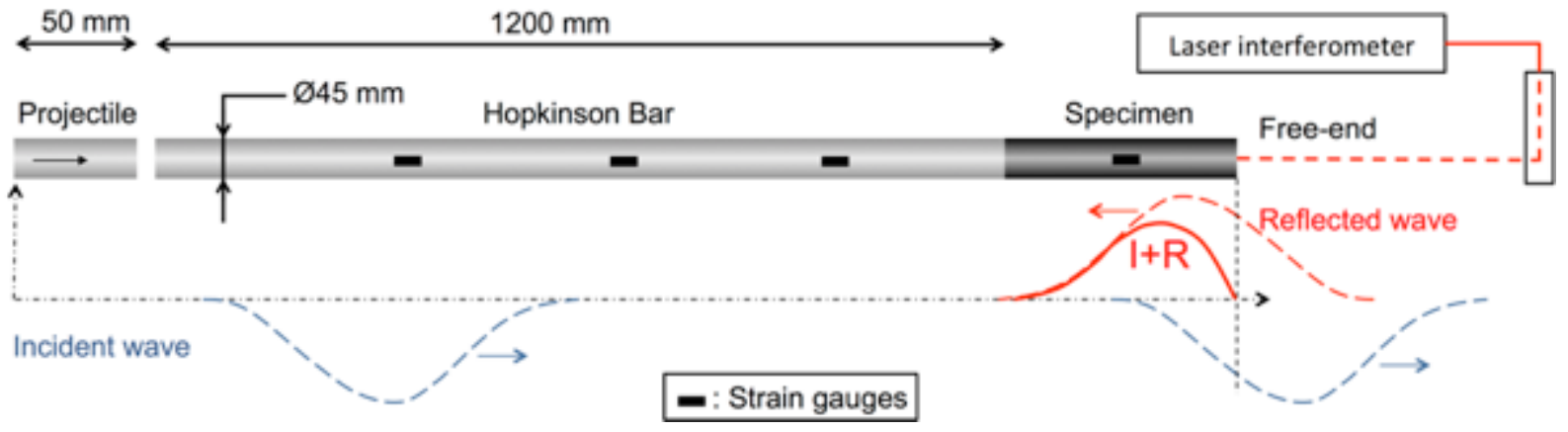

Figure 2: Experimental device for spalling tests. (The ultra-high-speed camera is not represented). 


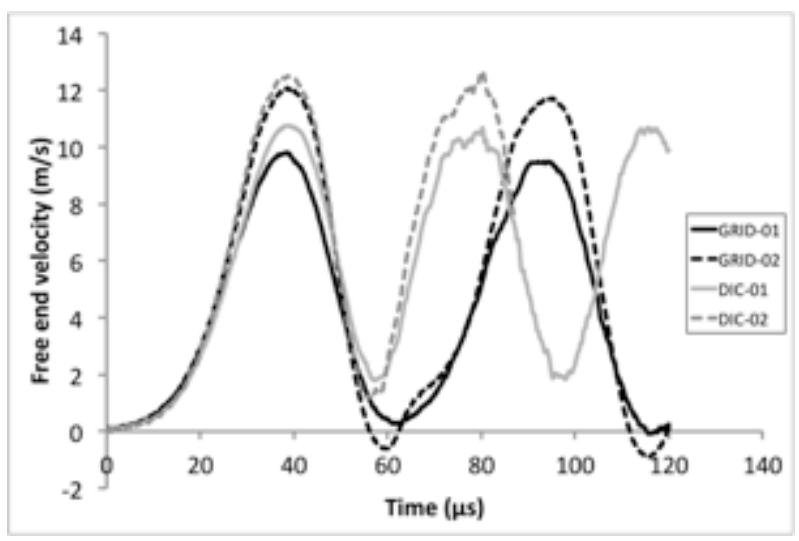

Figure 3: Velocity measured at the free-end of the specimen for the four tests. 


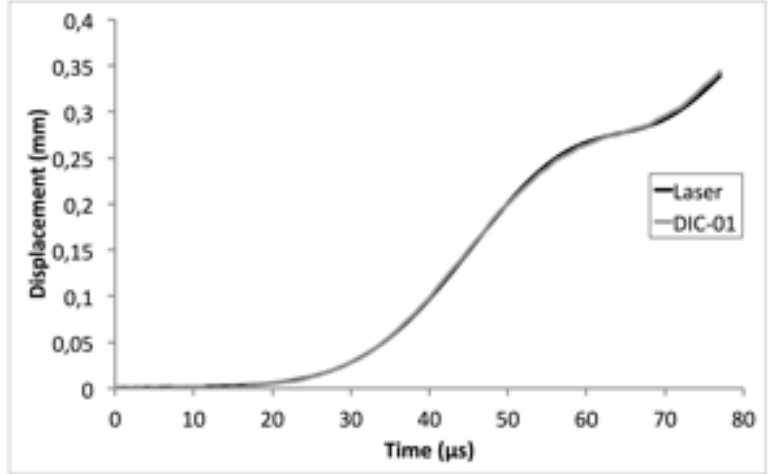

(a)

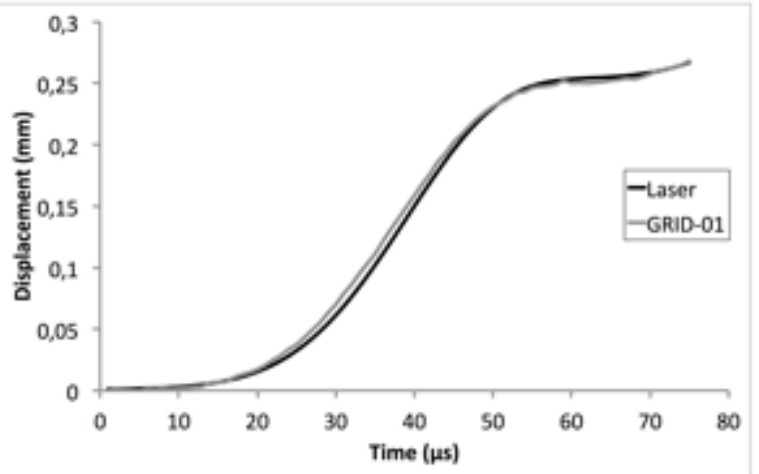

(c)

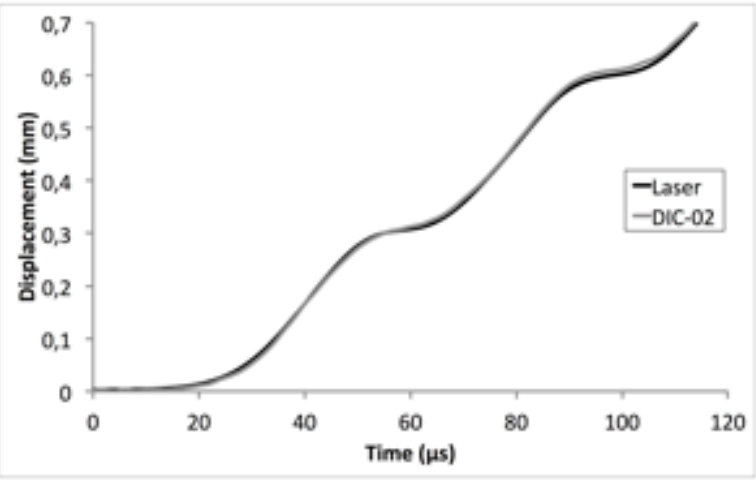

(b)

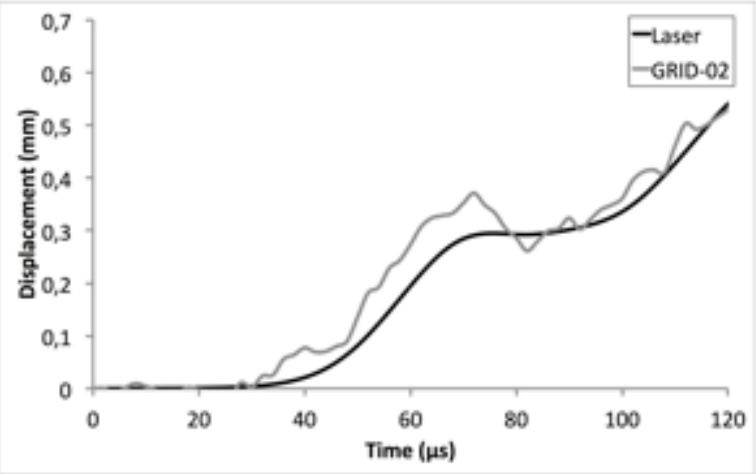

(d)

Figure 4: Displacement measurement with laser interferometer and the DIC (a, b) or the grid method (c, d). 


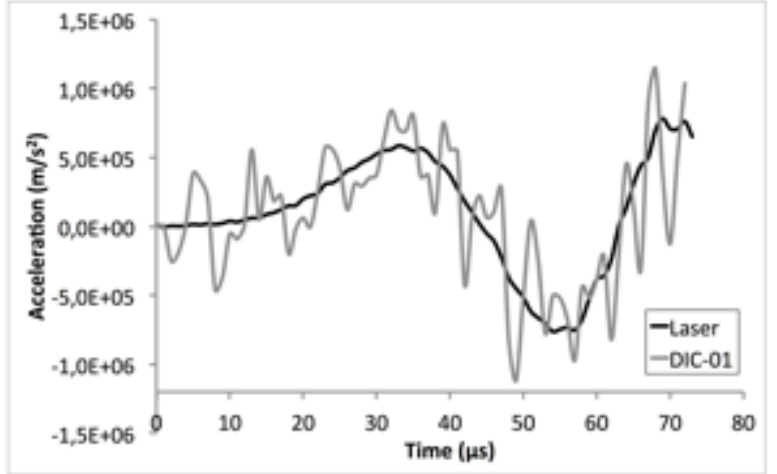

(a)

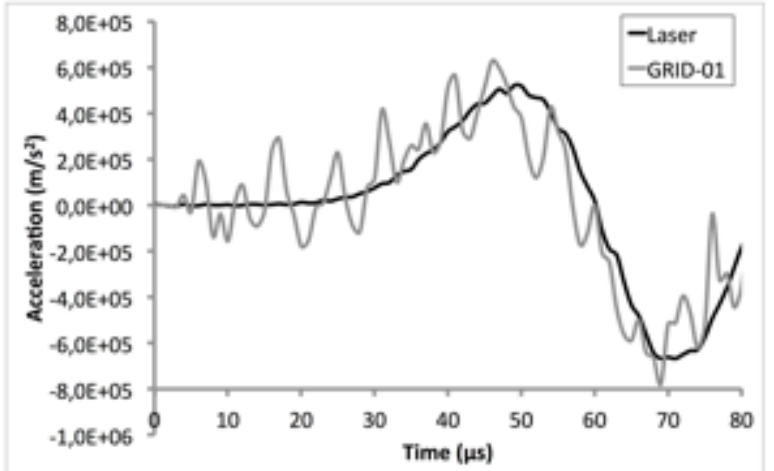

(c)

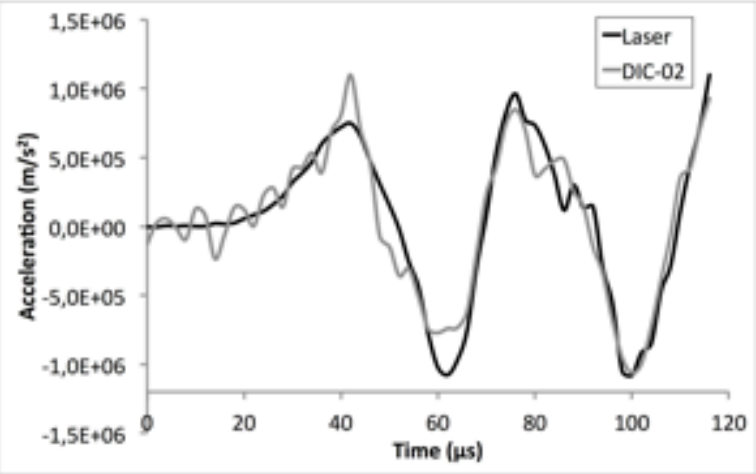

(b)

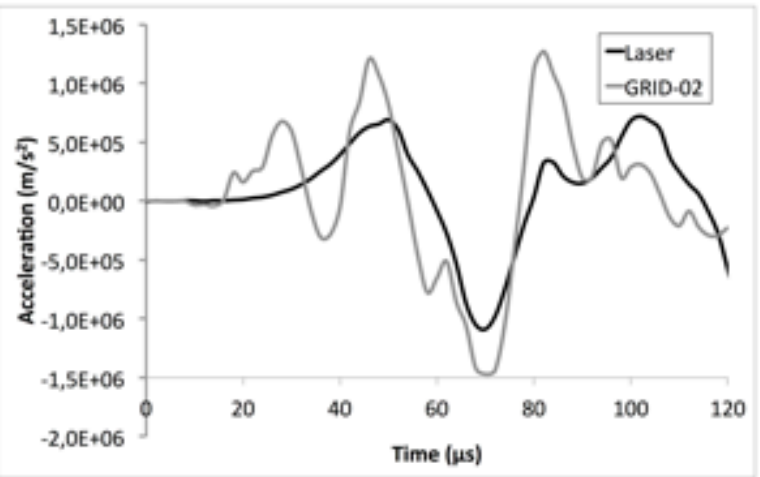

(d)

Figure 5: Acceleration measurement with laser interferometer and the DIC $(a, b)$ or the grid method (c, d). 


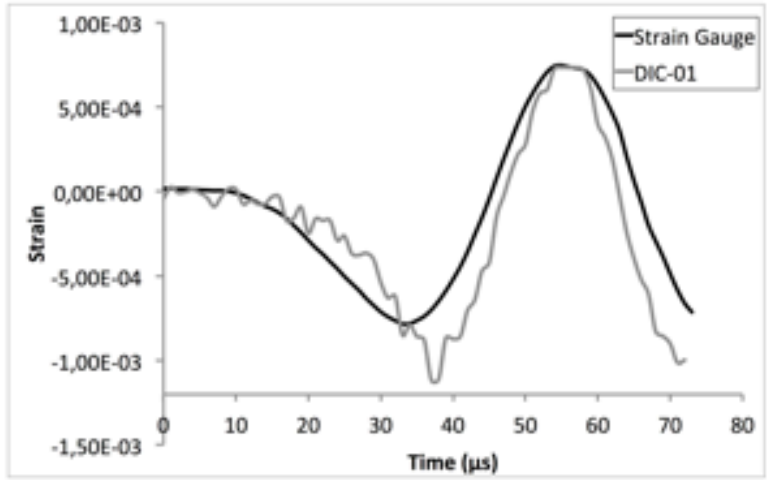

(a)

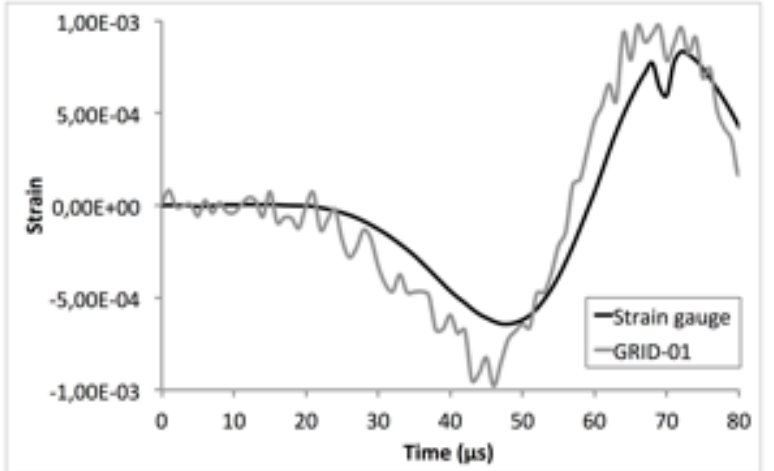

(c)

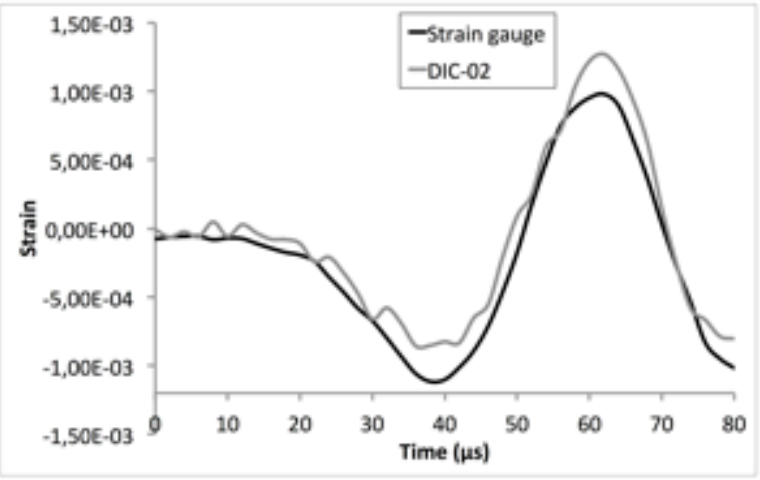

(b)

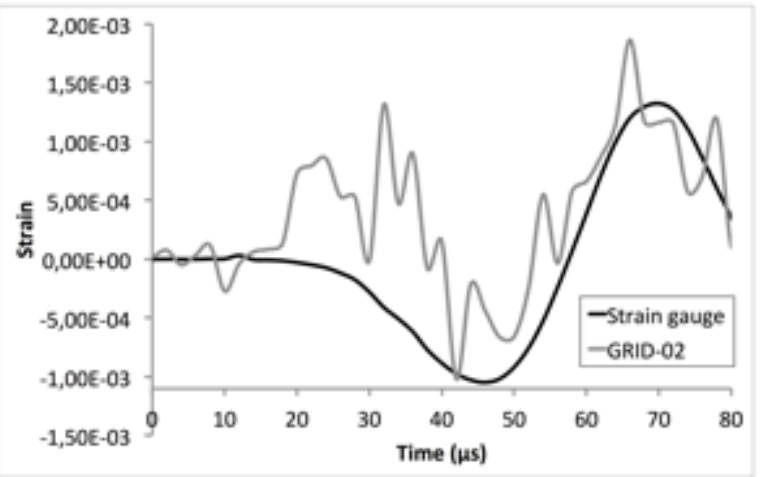

(d)

Figure 6: Strain measurement at $50 \mathrm{~mm}$ from the free-end of the specimen. Comparison between values from strain gauges and the DIC (a, b) or the grid methods (c, d). 


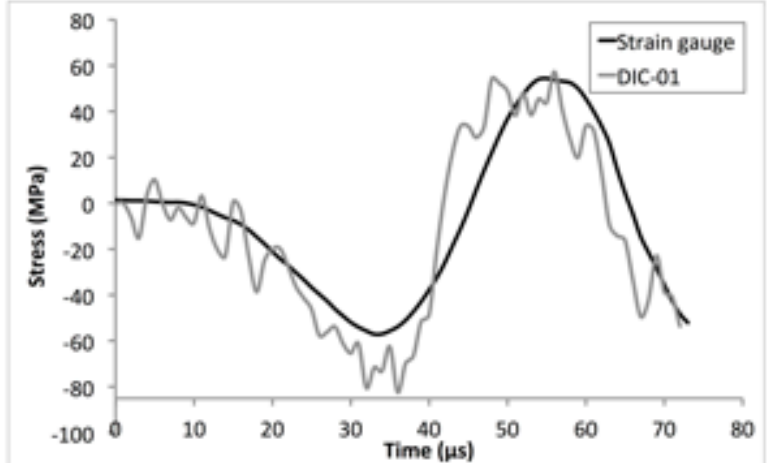

(a)

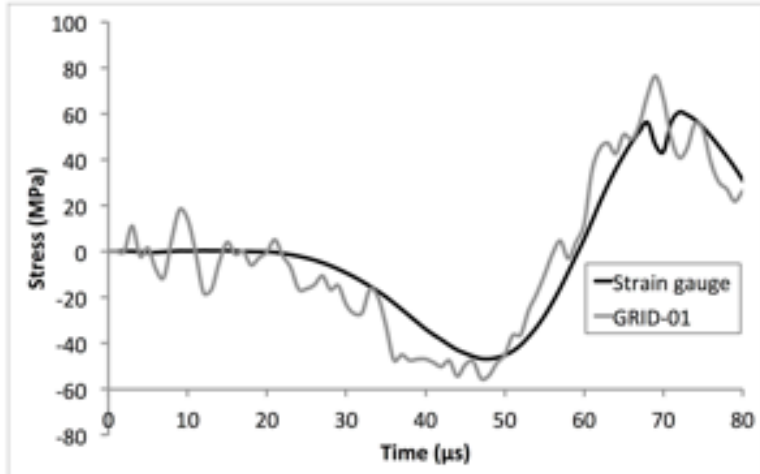

(c)

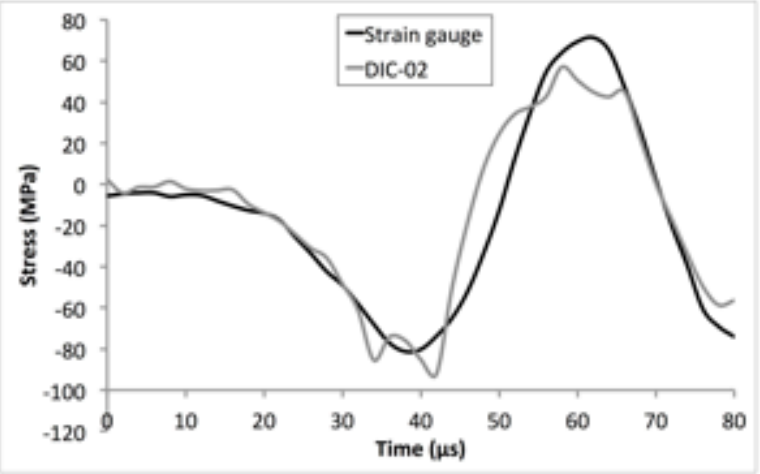

(b)

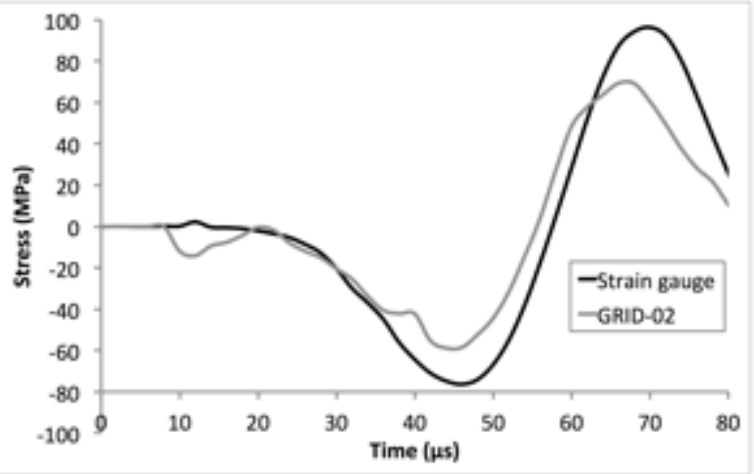

(d)

Figure 7: Stress measurement at $50 \mathrm{~mm}$ from the free-end of the specimen. Comparison between the strain gauge measurements and the one obtained with VFM associated to the DIC $(a, b)$ or the grid method (c, d). 


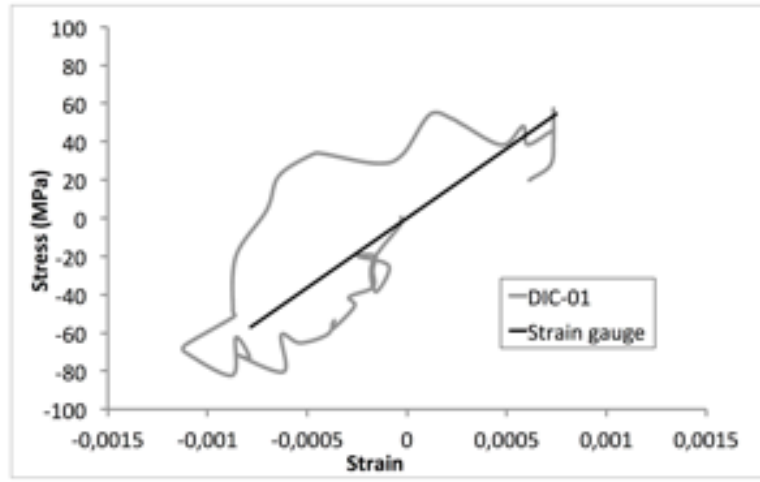

(a)

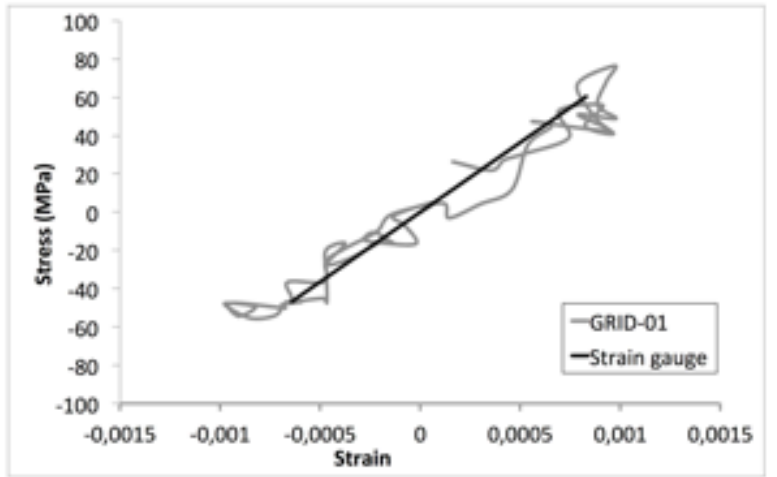

(c)

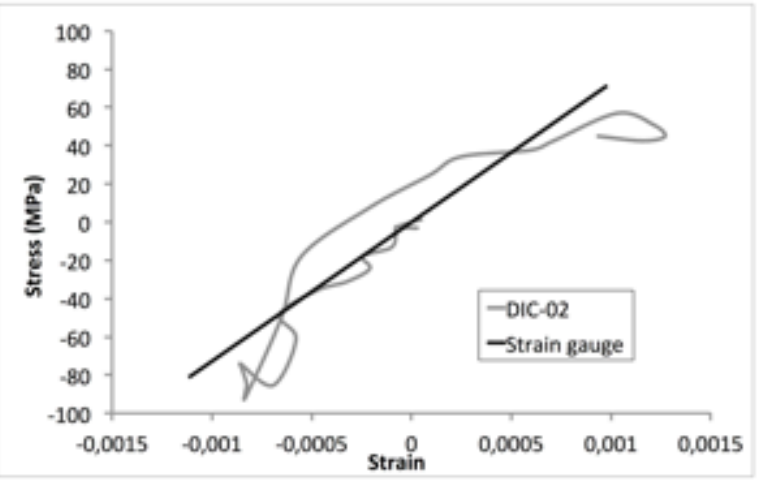

(b)

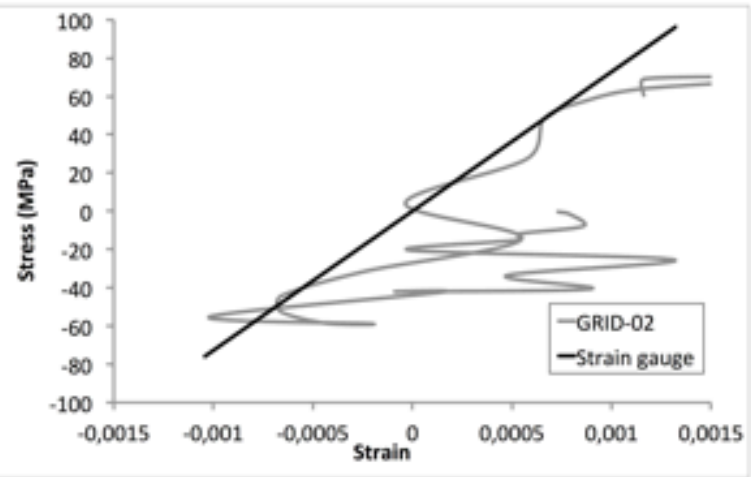

(d)

Figure 8: Strain-stress curves at $50 \mathrm{~mm}$ from the free-end of the specimen. Comparison between strain gauge measurements and the one obtained with VFM associated to DIC $(a, b)$ or grid method (c, d). 
Table 1 : Summary of the test campaign (DIC: Digital Image Correlation).

\begin{tabular}{|c|c|c|c|}
\hline Id. Test & $\begin{array}{c}\text { Frame rate } \\
\text { (fps) }\end{array}$ & $\begin{array}{c}\text { Image scale } \\
(\mathrm{mm} / \mathrm{px})\end{array}$ & $\begin{array}{c}\text { Measurement } \\
\text { method }\end{array}$ \\
\hline GRID-01 & $1^{\prime} 000^{\prime} 000$ & 0.2 & Grid \\
\hline GRID-02 & $500^{\prime} 000$ & 0.2 & Grid \\
\hline DIC-01 & $1^{\prime} 000$ '000 & 0.196 & DIC \\
\hline DIC-02 & 500 '000 & 0.196 & DIC \\
\hline
\end{tabular}

Table 2: Differences (\%) in the measurement of the displacement between the laser interferometer and DIC and grid techniques. (STD: standard deviation)

\begin{tabular}{|c|c|c|c|c|c|c|c|c|}
\hline Displacement & \multicolumn{2}{|c|}{ GRID-01 } & \multicolumn{2}{|c|}{ GRID-02 } & \multicolumn{2}{|c|}{ DIC-01 } & \multicolumn{2}{|c|}{ DIC-02 } \\
\hline $0.1 \mathrm{~mm}$ & \multicolumn{2}{|c|}{$8.9 \%$} & \multicolumn{2}{|c|}{$78.7 \%$} & \multicolumn{2}{|c|}{$1.2 \%$} & \multicolumn{2}{|c|}{$6.4 \%$} \\
\hline $0.2 \mathrm{~mm}$ & \multicolumn{2}{|c|}{$2.2 \%$} & \multicolumn{2}{|c|}{$40.4 \%$} & \multicolumn{2}{|c|}{$0.4 \%$} & \multicolumn{2}{|c|}{$1.2 \%$} \\
\hline Range & Mean & STD & Mean & STD & Mean & STD & Mean & STD \\
\hline $0.05-0.25 \mathrm{~mm}$ & $5.9 \%$ & $5.2 \%$ & $49.2 \%$ & $15.3 \%$ & $1.3 \%$ & $0.7 \%$ & $3.5 \%$ & $3.5 \%$ \\
\hline
\end{tabular}

Table 3: Differences (\%) in the measurement of the acceleration between the laser interferometer and DIC and grid techniques. (STD: standard deviation)

\begin{tabular}{|c|c|c|c|c|c|c|c|c|}
\hline & \multicolumn{2}{|c|}{ GRID-01 } & \multicolumn{2}{c|}{ GRID-02 } & \multicolumn{2}{c|}{ DIC-01 } & \multicolumn{2}{c|}{ DIC-02 } \\
\hline Phase & Mean & STD & Mean & STD & Mean & STD & Mean & STD \\
\hline Compression & $54.8 \%$ & $73.2 \%$ & $231 \%$ & $290 \%$ & $109 \%$ & $184 \%$ & $52.8 \%$ & $56.1 \%$ \\
\hline Tension & $221 \%$ & $713 \%$ & $378 \%$ & $236 \%$ & $648 \%$ & $2500 \%$ & $196 \%$ & $524 \%$ \\
\hline
\end{tabular}

Table 4 Differences (\%) in the measurement of the strain at $50 \mathrm{~mm}$ from the free-end of the specimen between the gauge and DIC and grid techniques. (STD: standard deviation)

\begin{tabular}{|c|c|c|c|c|c|c|c|c|}
\hline & \multicolumn{2}{|c|}{ GRID-01 } & \multicolumn{2}{c|}{ GRID-02 } & \multicolumn{2}{c|}{ DIC-01 } & \multicolumn{2}{c|}{ DIC-02 } \\
\hline Phase & Mean & STD & Mean & STD & Mean & STD & Mean & STD \\
\hline Compression & $64.2 \%$ & $55.7 \%$ & $167 \%$ & $160 \%$ & $51.6 \%$ & $43.6 \%$ & $32.6 \%$ & $35.4 \%$ \\
\hline Tension & $42.0 \%$ & $43.9 \%$ & $40.9 \%$ & $30.1 \%$ & $80.3 \%$ & $168 \%$ & $32.7 \%$ & $19.3 \%$ \\
\hline
\end{tabular}


Table 5: Difference (MPa) between the measurement of the stress at $50 \mathrm{~mm}$ from the free-end of the specimen between the gauge and DIC and grid techniques. (STD: standard deviation)

\begin{tabular}{|c|c|c|c|c|c|c|c|c|}
\hline & \multicolumn{2}{|c|}{ GRID-01 } & \multicolumn{2}{c|}{ GRID-02 } & \multicolumn{2}{c|}{ DIC-01 } & \multicolumn{2}{c|}{ DIC-02 } \\
\hline Phase & Mean & STD & Mean & STD & Mean & STD & Mean & STD \\
\hline Compression & $11.0 \mathrm{MPa}$ & $6.6 \mathrm{MPa}$ & $13.5 \mathrm{MPa}$ & $8.9 \mathrm{MPa}$ & $14.5 \mathrm{MPa}$ & $10.7 \mathrm{MPa}$ & $12.7 \mathrm{MPa}$ & $14.1 \mathrm{MPa}$ \\
\hline Tension & $12.3 \mathrm{MPa}$ & $8.2 \mathrm{MPa}$ & $23.3 \mathrm{MPa}$ & $9.6 \mathrm{MPa}$ & $16.8 \mathrm{MPa}$ & $10.2 \mathrm{MPa}$ & $13.0 \mathrm{MPa}$ & $9.5 \mathrm{MPa}$ \\
\hline
\end{tabular}

Table 6: Young modulus values identified with an ordinary least-squares evaluation.

\begin{tabular}{|l|l|l|l|}
\hline Id. Test & $\begin{array}{c}\text { Young Modulus } \\
\text { Identified (GPa) }\end{array}$ & R-square coefficient & $\begin{array}{l}\text { Difference with } \\
\text { Standard value }\end{array}$ \\
\hline DIC-01 & 65.6 & 0.6 & $9.8 \%$ \\
\hline DIC-02 & 63.6 & 0.86 & $12.6 \%$ \\
\hline GRID-01 & 62.2 & 0.95 & $14.6 \%$ \\
\hline GRID-02 & 46.2 & 0.56 & $36.5 \%$ \\
\hline
\end{tabular}

